\title{
Harnessing SAXS and X-ray crystallography for high-resolution structural studies of macromolecules
}

\author{
Miljan Simonović a \\ a Department of Biochemistry and Molecular Genetics, College of Medicine, The University of \\ Illinois at Chicago, Chicago, IL 60607, USA, msimon5@uic.edu
}

X-ray crystallography remains a dominant approach in high-resolution structural studies. However, because of the nature of the crystallization process and stringent requirements for homogeneity of crystalline matter to be analyzed by X-rays, the method provides a somewhat 'static' representation of a macromolecule under study. In addition, in the absence of any prior structural information and phase estimates, X-ray crystallography alone cannot provide even simplest descriptors about the target molecule structure and may fail to fully inform about structural changes in solution. Here, I will present a summary of our projects that studied enzymes responsible for accurate synthesis of human selenoproteins [1-3]. In all instances, X-ray crystallography and SAXS coupled to size-exclusion chromatography were pivotal for obtaining critical insights and successful completion of studies. Our results highlight the power of a synchronous application of X-ray crystallography and SAXS.

\section{References}

[1] French RL, Gupta N, Copeland PR, Simonović M (2014). J Biol Chem, 289, 28783-28794.

[2] Puppala AK, French RL, Mathies D, Baxa U, Subramaniam S, Simonović M (2016). Sci Reports 6 , 32563.

[3] Dobosz-Bartoszek M, Pinkerton MK, Otwinowski Z, Chakravarthy S, Söll D, Copeland PR, Simonović M (2016), Nature Commun 7, 12941. 